L1-5

\section{Psychological Effects of Disaster in Children} Kiwamu Tanaka, MD

Department of Psychiatry and Neurology, Kobe University School of Medicine, Kobe, Japan

1) Stress reactions in children

a) Acute phase

b) Restabilization phase

2) Children's experiences in a disaster
a) Destruction
b) Detachment
c) Bereavement
d) Confusion

3) Interventions
a) Children survivors
b) Family
c) Nurses and teachers

4) Expectations of mental health services

Keywords: children; disaster; effects; expectations; experiences; interventions; mental health; psychological; stress reactions

\section{L1-6}

Management for Alcohol-Related Problems After a Natural Disaster: In the Case of The Great HanshinAwaji Earthquake

Teteuro Noda, MD

Osaka Prefectural Mental Health and Welfare Center, Osaka City, Japan

Soon after the Great Hanshin-Awaji Earthquake, the concept of the post-traumatic stress disorder (PTSD) was introduced into Japan and immediately became popular. We believe the reason is that it is easy for people to understand PTSD as a very sympathetic mental illness that every person could affect all persons who suffer from traumatic events.

However, it actually was difficult for psychiatric staffs to manage the adjustment behavior of mentally ill patients at the shelters. Of special concern was alcoholism, as some alcoholics could drink with right reason because victims were likely to have the use of liquor recommended as a method for manage their stress. In the Japanese culture, people are permitted to drink for coping with sadness or pleasure on "hare"(unordinary days including feast and mourning days). After the earthquake, many bottles of liquor as material supports were sent to shelters. As it is estimated that almost $2 \%$ of Japanese are alcoholics, many treated and untreated alcoholics must have evacuated to shelters. It was very risky. The longer time after the earthquake, the more alcohol-related problems seemed to appear. Therefore, management for alcoholics gradually has become an important issue. Moreover, we worried about relapse of ex-alcoholics. Alcohol abstinence, self-help groups such as Danshukai and AA had come to have few meetings because every place where meetings could be held had become shelters.

Since victims had moved to temporary evacuation dwellings, it has begun to be recognized that many of the solitary deaths of living in single residences were associated with alcohol. Thus, after the earthquake, alcohol-related problems have been continuous and serious.

This presentation will include: 1) Preventive strategy for alcohol-related problems; and 2) Method for supporting alcoholics at shelters and temporary evacuation dwellings.

Keywords: alcohol; alcoholism; culture; disasters; Great HanshinAwaji Earthquake; management; mental illness; post-traumatic stress disorder (PTSD); psychiatry; self-help; shelters; support

\section{General Session XIII Disaster Epidemiology Tuesday, 11 May, 8:30-9:45 hours Chair: M. Wayne Greene, Hiroshi Henmi}

G-62

The Risk Factors of Crush Syndrome Patients for Renal Failure, Hemodialysis, and Death

Noriaki Aoki, $M D ;_{1,1}^{1,2}$ Ernesto A. Pretto, $M D ;^{3}$

Jun Oda, $M D{ }^{2}$ Katsubiko Sugimoto $M D ;^{4}$

J. Robert Beck, $M D ;{ }^{1}$ Hiroshi Tanaka, $M D ;^{2}$

Tosbibaru Yosbioka, $M D ;^{2}$ Tsuguya Fukui, $M D^{2}$

1. Information Technology Program, Baylor College of Medicine, USA

2. Department of General Medicine and Clinical Epidemiology, Kyoto University Hospital, Kyoto, Japan

3. Safar Center for Resuscitation Research, University of Pittsburgh, Pittsburgh, Pennsylvania USA

4. Department of Emergency and Critical Care Medicine, Showa University Hospital, Tokyo, Japan

5. Department of Traumatology and Emergency Medicine, Osaka University Medical School, Osaka, Japan

Introduction: Although numerous reports have described crush syndrome patients after a large earthquake, risk factors for severe complication and a deleterious outcome never have been established because of scarcity of epidemiological and quantitative analysis. In this study, we clarify the risk factors and the effectiveness of fluid resuscitation for crush syndrome.

Methods: We used data from 372 crush syndrome patients reported by Oda et al. Three outcomes were employed: 1) renal failure (RF) defined as serum creatinine $(\mathrm{Cr})>2.5 \mathrm{mg} / \mathrm{dl} ; 2$ ) induced hemodialysis (HD); and 3) death. A total of 21 risk factors were grouped into seven domains: 1) patients characteristics; 2) physical examination and laboratory data without specific device; 3) laboratory data using specific devices; 4) life-saving effort; 5) severity of crush syndrome; 6) therapeutic approach; and 7) the site of injury. Logistic regression models were used to control for confounding variables and to assess interaction between variables after a univariate analysis for all 21 variables. The Mantel-Haenstzel test was used to assess the effects of volume resuscitation therapy stratified by severity of crush syndrome. In all tests, a $p$-value of 0.05 or less was considered to be statistically significant. 